Illinois State University

ISU ReD: Research and eData

Theses and Dissertations

$4-5-2018$

\title{
Supporting the Medial Longitudinal Arch: A Comparison Between Intrinsic and Extrinsic Musculature
}

James D. Schaefer

Illinois State University, jdschae1@ilstu.edu

Follow this and additional works at: https://ir.library.illinoisstate.edu/etd

Part of the Kinesiology Commons

\section{Recommended Citation}

Schaefer, James D., "Supporting the Medial Longitudinal Arch: A Comparison Between Intrinsic and Extrinsic Musculature" (2018). Theses and Dissertations. 884.

https://ir.library.illinoisstate.edu/etd/884

This Thesis is brought to you for free and open access by ISU ReD: Research and eData. It has been accepted for inclusion in Theses and Dissertations by an authorized administrator of ISU ReD: Research and eData. For more information, please contact ISUReD@ilstu.edu. 


\section{SUPPORTING THE MEDIAL LONGITUDINAL ARCH: A COMPARISON BETWEEN INTRINSIC AND EXTRINSIC MUSCULATURE}

\section{JAMES D. SCHAEFER}

\section{Pages}

Context: Considerable research has been done to study what muscles best support the medial longitudinal arch (MLA). However these studies look at intrinsic or extrinsic muscles individually rather than comparing their effects at support of the MLA in a static stance. Researchers have yet to examine the changes to the MLA in the gait cycle rather than just looking at it from a static point of view. Objective: To study the effectiveness of two strengthening protocols for supporting the medial longitudinal arch during stance and gait.

Design: Single-blinded, randomized control trial. Setting: Testing was completed in two athletic training facilities. Patients or Other Participants: A total of 24 recreationally active patients (14 females, 10 males) participated. Interventions: Individual strengthening protocols for intrinsic and extrinsic muscles respectively. Main Outcome Measure(s): Static measurements of navicular drop. Dynamic measurement of plantar pressure measuring contact area in square centimeters of the midfoot. To compare the effects of the intervention, two, one-way ANOVAs were used to compare change scores for the 3 intervention groups. Results: A significant difference between groups was found for the change in navicular drop $(\mathrm{p}=0.001)$, but not plantar pressure area $(\mathrm{p}=0.37)$. Post hoc comparisons for the change in navicular drop revealed a significant difference between the extrinsic and control group $(\mathrm{p}=0.001$, effect size $=2.15,95 \%$ $\mathrm{CI}=0.92$ to 3.38 ) and the extrinsic and intrinsic group ( $\mathrm{p}=0.03$, effect $\operatorname{size}=1.31,95 \% \mathrm{CI}=0.23$ to 
2.39), but no difference between the control and intrinsic group $(\mathrm{p}=0.31)$. Conclusions: These results appear to demonstrate that extrinsic muscles of the foot have a greater effect in support of the medial longitudinal arch during static stance. However, when dynamic measurements of plantar pressures were measured, there were no significant results noted for either intervention group. These results suggest that static standing exercises have no effect on dynamic support on the medial longitudinal arch of the foot. This can lead to future research to study what specifically causes dynamic changes of foot posture to occur.

KEYWORDS: intrinsic foot muscles; extrinsic foot muscles; medial longitudinal arch; pes planus 
SUPPORTING THE MEDIAL LONGITUDINAL ARCH: A COMPARISON BETWEEN

INTRINSIC AND EXTRINSIC MUSCULATURE

JAMES D. SCHAEFER

A Thesis Submitted in Partial

Fulfillment of the Requirements

for the Degree of

MASTER OF SCIENCE

School of Kinesiology and Recreation

ILLINOIS STATE UNIVERSITY

2018 
Copyright 2018 James D. Schaefer 


\section{SUPPORTING THE MEDIAL LONGITUDINAL ARCH: A COMPARISON BETWEEN INTRINSIC AND EXTRINSIC MUSCULATURE}

JAMES D. SCHAEFER

COMMITTEE MEMBERS:

Justin Stanek, Chair

Adam Jagodinsky 


\section{ACKNOWLEDGMENTS}

I would like to thank my committee formed by Dr. Justin Stanek and Dr. Adam

Jagodinsky. I appreciate your guidance through this process and the time you have given to help me complete this study. I would like to thank Bryce Brown for being my research assistant.

J. D. S. 


\section{CONTENTS}

Page

ACKNOWLEDGMENTS

TABLES

CHAPTER I: INTRODUCTION 1

CHAPTER II: REVIEW OF LITERATURE

Bony Anatomy $\quad 4$

$\begin{array}{ll}\text { Arches of the Foot } & 7\end{array}$

What Supports These Arches? $\quad 8$

Passive and active support of the longitudinal arches. $\quad 8$

Intrinsic foot muscles. $\quad 9$

$\begin{array}{ll}\text { Tibialis posterior. } & 12\end{array}$

$\begin{array}{ll}\text { Arch Deformities } & 13\end{array}$

Calcaneal Eversion in Relationship to Pronation 15

Treatment of Pes Planus 16

Intrinsic muscle strengthening/effects on navicular drop. 16

$\begin{array}{lr}\text { Tibialis posterior strengthening. } & 18\end{array}$

$\begin{array}{ll}\text { Classification and Measurement } & 19\end{array}$

$\begin{array}{ll}\text { Arch indices. } & 20\end{array}$

Navicular drop test. $\quad 21$

$\begin{array}{ll}\text { Plantar pressures. } & 23\end{array}$

CHAPTER III: METHODOLOGY 25

$\begin{array}{ll}\text { Participants } & 25\end{array}$ 
Instrumentation

$\begin{array}{ll}\text { Procedures } & 26\end{array}$

$\begin{array}{ll}\text { Intervention } & 28\end{array}$

$\begin{array}{ll}\text { Intrinsic muscle strengthening. } & 29\end{array}$

$\begin{array}{ll}\text { Extrinsic muscle strengthening. } & 30\end{array}$

CHAPTER IV: RESULTS

$\begin{array}{ll}\text { Statistical Analysis } & 32\end{array}$

$\begin{array}{ll}\text { Results } & 32\end{array}$

CHAPTER V: DISCUSSION AND CONLCUSION 35

$\begin{array}{ll}\text { Discussion } & 35\end{array}$

$\begin{array}{ll}\text { Limitations } & 38\end{array}$

$\begin{array}{ll}\text { Conclusions and Future Research } & 38\end{array}$

$\begin{array}{ll}\text { REFERENCES } & 39\end{array}$

APPENDIX A: EXERCISE HANDOUT 53 


\section{TABLES}

Table

Page

1. Descriptive Statistics for Demographic Data by Group 33

2. Descriptive Statistics for Navicular Drop, Arch Height Index and Plantar-Pressure

3. Effect Size for Group Differences Pre to Post Intervention of Navicular Drop

4. Effect Size for Between Group Differences Post Intervention 


\section{CHAPTER I: INTRODUCTION}

Support of the medial longitudinal arch (MLA) is important for the foot's ability to maintain proper foot posture, and efficiently transmit forces up the lower kinetic chain. Maintaining strength of the intrinsic and extrinsic foot musculature can help in supporting this pivotal structure of the foot. Dysfunction of the MLA is shown to have an association with injuries of the ankle, tibia, knee, and hip. ${ }^{1-4}$ A small dynamic change in the foot can cause several compensations up the lower kinetic chain. The architecture and musculature of the foot has evolved overtime as the demands of the body place on it have changed. ${ }^{3}$ The MLA acts as a mechanism to absorb forces while in weight bearing to reduce forces transmitted up the lower leg. ${ }^{1}$ The MLA can be classified based on the height of the arch itself; pes planus, pes cavus, and recurvatum (rocker bottom foot) are the three common, and most basic, clinical classifications. ${ }^{1}$ Characterized by excessively pronated feet, pes planus is the most common foot problem in adult individuals in the United States and can be measured by assessing how much the height of the MLA drops when an increase of load is applied., ${ }^{2,5}$

Extrinsic muscles of the foot have been thought to have a substantial effect on supporting MLA during dynamic movements. ${ }^{5,6}$ One specific muscle that receives a lot of attention is the tibialis posterior (TP) which has multiple insertions once it passes the medial malleolus and enters into the foot. ${ }^{7}$ The TP is the primary extrinsic muscle of the foot that is thought to support the medial longitudinal arch. ${ }^{6-10}$ The complex anatomy of the TP insertion sites serve to support the MLA and dysfunction of this muscle can lead to decreased stability of the MLA and an increase in likelihood of flat foot deformity (FFD). ${ }^{11}$ The TP tendon and the spring ligament are stretched in individuals with FFD, decreasing the ability of the dynamic and static stabilizers to 
function properly and causing a lowering in the height of the MLA. ${ }^{1}$ Research supports the idea that the TP plays a vital role in support of the MLA during cyclic loading. ${ }^{8}$

The intrinsic muscles (IM) of the foot are also often cited as providing the primary support for the MLA. ${ }^{12}$ These muscles both originate and insert within the foot and have small moment arms, thus generating only a small amount of force. ${ }^{3}$ A foot core system has been proposed to draw an analogy between the body's core stabilizers and the IM that stabilize the foot. ${ }^{3}$ The idea of the foot core is that proper function of the intrinsic stabilizers provide a stable support for prime movers to function properly and produce gross movements. IM of the foot act as the stable base for prime extrinsic muscles such as the TP to perform the gross movements of the ankle. Activation of IM is shown to increase as postural demand increases such as when an individual is in full weight bearing. ${ }^{13}$ This data supports the idea of IM being important to helping the TP function during dynamic movement. Theoretically, increased IM activation during weight bearing should effect MLA height. Active muscles will contract and pull the metatarsal heads posterior towards the calcaneus which will increase the MLA height. When the IM fatigue this causes a significant decrease in the MLA height. ${ }^{14}$ Exercises to strengthen the IM causes a shortening of the MLA suggesting that short foot exercises (SFE) can help increase height of MLA. ${ }^{15}$

The intrinsic and extrinsic muscles have been shown to support the MLA and in some cases increase the height of the MLA with proper strengthening protocols. Researchers have studied various ways to measure the height of the MLA in direct and indirect ways. ${ }^{16-18}$ The navicular drop test has been used as well as the arch height index, with both having sufficient reliability ${ }^{19,20}$ The arch height index measures from the head of the first metatarsal and most posterior aspect of the calcaneus. This length is combined with the height of the dorsum of the 
foot and the ratio is use to classify height of the MLA as a measure of foot posture. ${ }^{21}$ Studies continue to look at extrinsic and intrinsic foot musculature separately rather than together. To the researcher's knowledge there has been no study done comparing effect of strengthening of intrinsic muscles against extrinsic muscles and which one is more equipped to support the MLA. The purpose of this study was to use a strengthening protocol specifically designed to improve strength of intrinsic muscles and compare to a strengthening protocol for the extrinsic muscles to see which muscle group better supports the MLA. We hypothesize that the height of the medial longitudinal arch will increase and the average plantar pressure of the midfoot will decrease as a result of strengthening both intrinsic and extrinsic muscles of the foot. 


\section{CHAPTER II: REVIEW OF LITERATURE}

The ankle and foot have a unique relationship with the lower extremity. It serves as the base of the kinetic chain that transfers forces up the lower extremity. ${ }^{22}$ There is many different muscles, ligaments and fascia that support and move the foot and ankle. Breaking it down there are 28 bones, 33 joints, 112 ligaments, 13 extrinsic muscles and 21 intrinsic muscles. ${ }^{18}$ Specifically looking at the arches of the foot there are extrinsic and intrinsic factors that support the longitudinal and transverse arches of the foot. Understanding the anatomy and function of the foot leads to a better understanding of how to strengthen and support the various foot structures. The purpose of this research is to identify what specific treatment protocol best increases height of the medial longitudinal arch by way of muscle strengthening. Arch deformities are prevalent among the general population and various treatment methods are instituted to combat both arch deformity and the ancillary issues it causes up the kinetic chain. What is the best treatment method regarding strengthening intrinsic muscles or extrinsic muscles.

\section{Bony Anatomy}

When studying the foot and specifically the medial longitudinal arch you must first think of the entire lower kinetic chain. How the body transmits ground reaction forces and forces produced by muscles up and down this chain effects the biomechanics of the lower extremity and more importantly the kinematics of the foot. The lower leg is made up of two bones which are the tibia and the fibula. At its proximal end, the narrow fibula has a head that holds an articulating surface for the synovial joint between itself and the tibia. The majority of the shaft of the fibula holds attachment sites for muscles; however a medial edge holds attachment to the interosseous membrane which helps attach the fibula to the tibia. These attaching tissues provide dynamic support for various movements. At the distal end of the fibula is a bony prominence 
called the lateral malleolus, this forms the lateral aspect of the ankle joint. The proximal aspect of the tibia which is the larger of the two bones in the lower leg has an articular surface with the distal end of the femur and the medial and lateral femoral condyles. On the posterior aspect of the lateral femoral condyle there is an articulation with the proximal head of the fibula. Distally the tibia begins to flare into a larger diameter to form the medial malleolus. On the lateral surface of the malleolus is an articular surface that forms the medial aspect of the ankle joint. The inferior articular surface of the distal tibia transmits forces from the foot to the lower leg as well as the tibia onto the foot. The distal end of the tibia and fibula form the mortis that makes up the talocrural joint. This syndesmotic joint is supported by tibiofibular ligaments as well as the interosseous membrane. ${ }^{23}$ Sagittal plane movements occur at the talocrural joint with dorsiflexion and plantarflexion. The subtalar joint just inferior to the talus is where frontal plane movement occurs in the form of ankle inversion and eversion. The most distal aspect of the lower leg is the foot. The foot is comprised of 26 total bones made up of seven tarsals, 5 metatarsals, and 14 phalanges. The tarsals make up the hind foot and the metatarsals and phalanges form what is referred to as the forefoot. The main bony connections between the foot and the lower leg are the talus. The talus rests superior to the calcaneus while the talus articulates with the navicular, the calcaneus articulates with the cuboid. On the medial aspect of the foot just distal to the navicular are the medial, intermediate, and lateral cuneiforms. Distal to the tarsals are the metatarsals and the phalanges. ${ }^{23}$ The alignment of the bony anatomy of the foot formed by the tarsals, metatarsals and ligaments produces the formation of the transverse and longitudinal arches. The two longitudinal arches are the lateral and medial longitudinal arch. ${ }^{1}$ The supporting arches are designed to absorb and distribute forces during movement as well as improve locomotion by propelling the body forwards during gait. ${ }^{1}$ 
Diving deeper into the bony anatomy of the foot we see the various landmarks and articulations that allow the foot to perform various functions. Moving proximal to distal, we begin with the talus which articulates with the inferior surface of the tibia, the medial surface of the lateral malleolus, lateral surface of the medial malleolus, the calcaneus, and the head of the navicular. The talus is wide at the front and narrow at the back. The narrowness of the back of the talus allows for increased inversion and eversion when the ankle is in a loose packed position such as plantar flexion. The closed packed position of dorsiflexion pushes the wide head of the talus into the mortis joint which creates more stability at the ankle. Just inferior to the talus lay the calcaneus which forms the heel of the foot. The calcaneal tuberosity has medial and lateral processes that support the weight transmitted to the heel. ${ }^{23}$ The superior aspect of the calcaneus has articular facets for the talus and the largest surface projecting off of medial aspect of the calcaneus is the sustentaculum tali. The last articulation of the calcaneus is with the cuboid bone. The cuboid not only articulates with the calcaneus, but also has articulations with the fourth and fifth metatarsals. There is also a small a small medial articulation with the lateral cuneiform. The navicular sits distal to the talus and medial to the cuboid. The distal articulations are with the medial, intermediate and lateral cuneiforms. The medial bony prominence is the navicular tuberosity which is easily palpable inferior and distal to the medial malleolus. The three cuneiforms have proximal articulations with the navicular and the lateral cuneiform articulates with the cuboid. All of these articulations are through synovial joints. The three cuneiforms articulate with the first through third bases of the metatarsals. The metatarsals are similar to the metacarpals in the hand. They are comprised of a proximal base, the shaft, and the distal head. The phalanges are similar in description to the metatarsals. Each metatarsal base articulates with 
the one next to it and the heads articulate with the phalanges distal to them. Two sesamoid bones are commonly seen on the inferior aspect of the metatarsal phalangeal joint of the first toe..$^{23}$

\section{Arches of the Foot}

The foot has three separate arches consisting of twelve total bones from the calcaneus to the metatarsals. ${ }^{24}$ These arches are designed to absorb and distribute body weight, providing both stability and flexibility during various phases of movement. The arches of the foot provide an elastic connection between the forefoot and the hind foot. ${ }^{1}$ They also provide our base of support during standing; however, during movement the arches allow the foot to attenuate loads. These arches own spring-like characteristics that store and release energy with each foot strike. Evolutionary research suggests that the foot arch has developed in response to the increased demands of walking and running. The stability of the arch is thought to be the central core of the foot and is imperative to normal foot function. ${ }^{3}$ This unique relationship helps by ensuring that the majority of forces confronted during weight bearing can be dissipated before reaching the bones of the lower leg. ${ }^{1,23}$ There are two longitudinal arches that run medial and lateral as well as a transverse arch that is anatomically located in the frontal plane. The transverse arch is formed by the head of the talus, the navicular, calcaneus, cuboid and the base of the metatarsals. ${ }^{23}$ The intermediate cuneiform serves as the keystone of the transverse arch. ${ }^{1,25}$ As the transverse arch moves distally down the foot it begins to flatten out and the heads of the metatarsals are all on the same plane and share the duties of weight bearing. ${ }^{23}$ The lateral longitudinal arch begins at the calcaneus and travels through the cuboid, to the heads of the fourth and fifth metatarsals. ${ }^{23}$ With the cuboid being the most integral part of the lateral longitudinal arch. ${ }^{26}$ Stresses on the arches are not proportional and the medial longitudinal arch is viewed as the most significant clinically when studying flat foot deformities. ${ }^{1,23}$ The medial longitudinal arch supports the 
body-weight loaded from the talus up to the tibia. ${ }^{24}$ The medial longitudinal arch starts with the calcaneus and moves distal through the talus, navicular, cuneiforms, and to the heads of the first, second and third metatarsals. ${ }^{23,24}$ The navicular, which is an attachment site for several soft tissue structures, serves as the key bony component to the medial longitudinal arch. ${ }^{25}$ Normally the medial longitudinal arch has three classifications of normal, low, and high. ${ }^{1,27}$ The low arch, or "pes planus" can lead to less optimal foot function and is referenced with the development of lower extremity and back injuries. ${ }^{27-30}$ Pes planus affects the ability of the bones of the foot to lock and form a ridged lever during walking. The lever allows for propulsion and absorption of forces. ${ }^{1}$ Even though these arches are defined separately, it has been proposed that the arches blend together into a half dome responsible for adapting to load changes during static and dynamic activities. ${ }^{31}$

\section{What Supports These Arches?}

Passive and active support of the longitudinal arches. The medial longitudinal arch is supported both passively and actively by ligaments, plantar aponeurosis, and muscles. ${ }^{8}$ The main static supports of the medial longitudinal arch are the plantar aponeurosis, the long and short plantar ligaments and the plantar calcanonavicular ligament, also known as the spring ligament. ${ }^{8,32}$ Huang et al. ${ }^{33}$ found that the highest static contribution to arch stability is provided by the plantar aponeurosis. The spring ligament has two main components, the superomedial and the inferior calcanonavicular ligament. ${ }^{34}$ The superomedial portion is medial to the talar head and is the portion that blends with the deltoid ligament. ${ }^{33-37}$ The calcanonavicular portion originates from the sustentaculum tali on the medial aspect of the calcaneus and inserts on the most prominent medial aspect of the navicular tubercle. ${ }^{1,23}$ As the ligament spans the proximal third of the medial aspect of the foot it creates a sling for the lower surface of the head of the talus to 
rest. ${ }^{23}$ The spring ligament restricts joint motion that contributes to the flattening of the arch. It also adds some elasticity to the arch which allows the arch to return to its unloaded state when not bearing weight. ${ }^{1,23}$ The lateral longitudinal arch is supported by the long plantar ligament which originates on the lateral aspect of the calcaneus posteriorly and inserts on the cuboid and the third through fifth metatarsals. ${ }^{23}$ The plantar aponeurosis or plantar fascia has a primary function to provide rigid support of the arches during the propulsion phase of gait, this is done through the Windlass effect. ${ }^{38}$ The Windlass effect occurs when the great toe moves into extension and provides a tension on the plantar aponeurosis. This shortens the fascia and supports the medial longitudinal arch.

Active support of the medial longitudinal arch comes from both intrinsic and extrinsic musculature including the anterior and posterior tibialis, fibularis longus and the plantar foot intrinsic muscles. Of the intrinsic muscles the abductor hallucis, the flexor digitorum brevis and the quadratus plantae are three muscles thought to contribute the most support. ${ }^{39}$ However, surface EMG does not allow the examination of the deeper layers of the plantar intrinsic muscles. ${ }^{13,14,40}$

Intrinsic foot muscles. Several muscles within the foot contribute to the support of the medial longitudinal arch. ${ }^{12,41,42}$ These muscles have small moment arms, small cross-sectional areas and do not produce a high magnitude of force. ${ }^{3}$ Muscles within the foot are termed to be intrinsic, defined by having both an origin and insertion within the foot without crossing the ankle joint. ${ }^{43,44}$ Intrinsic foot muscles have origins and insertions on both the dorsal and plantar aspect of the foot. The plantar intrinsic muscles contribute more to support of the arches of the foot relative to the dorsal muscles. Allen and Gross ${ }^{12}$ suggested a theory that the weak intrinsic muscles provide insufficient dynamic support to the medial longitudinal arch which causes and 
increased strain on the plantar aponeurosis. This is supported by Headlee et al. who found that significantly fatiguing the intrinsic muscles of the foot increases navicular drop. ${ }^{14}$

The plantar intrinsic foot muscles are organized into four layers with the most superficial layer being deep to the plantar aponeurosis. ${ }^{3,43,44}$ The first two layers have muscle configurations that align with the medial and lateral longitudinal arches of the foot, whereas the deeper layers align more so with the anterior and posterior transverse arches. ${ }^{3}$ Composed of the abductor hallucis, flexor digitorum brevis, and the abductor digiti minimi ${ }^{43}$ The abductor hallucis is the most medial muscle in the first layer. This muscle originates from the posteromedial calcaneus and inserts into the medial sesamoid on the plantar aspect of the first ray ${ }^{45}$ Wong et al. ${ }^{46}$ theorize that the abductor hallucis muscle is active during abduction of the hallucis, flexion and supination of the first metatarsal, inversion of the calcaneus and external rotation of the tibia in combination with the elevation of the medial longitudinal arch. The abductor hallucis muscle plays an important role supporting the medial longitudinal arch and resisting pronation in static stance. ${ }^{14,40}$ The second layer is made up of the quadtratus plantae and the lumbricals. The third layer consists of adductor hallucis transverse, adductor hallucis oblique, flexor hallucis brevis and flexor digit minimi brevis. The deepest layer is comprised of three plantar interossei. All the plantar intrinsic muscles share an innervation from the medial and lateral plantar branches of the tibial nerve. ${ }^{44}$ Research using electromyography shows small amounts of activity in the abductor halluces, flexor digitorum brevis and the quadratus plantae muscles during standing. These muscle signals increase with increased postural demands, such as increase body mass or weight being carried. ${ }^{3,13}$ Hashimoto et al. found that increases in intrinsic foot flexor strength lead to shorter longitudinal and horizontal foot arches. Other studies show that the abductor halluces, flexor halluces brevis, flexor digitorum brevis, and interosseous muscles help stabilize the foot 
arch during propulsion. Making the foot ridged during toe off rather than loose when in standing position. $^{47-49}$

The intrinsic muscles on the dorsum of the foot are divided into two layers. ${ }^{43}$ The dorsal intrinsic muscles do not appear in much literature and their function is relatively unknown in relation to the plantar intrinsic muscles. ${ }^{50}$ The most superficial layer houses the extensor hallucis brevis and extensor digitorum brevis. These two muscles are innervated by the deep fibular nerve. The second and most deep layer holds the dorsal interossei muscles and is innervated by the lateral plantar nerve with the first and second dorsal interossei also receiving part of their innervation from the deep fibular nerve. ${ }^{44}$

Intrinsic foot muscles contribute an important role in supporting the medial longitudinal arch during gait, however studies also show that there is muscle activation during static standing as well. ${ }^{13,49}$ These muscle signals increase with increase in postural loading and there is also evidence that weakness in the plantar intrinsic muscles has been implicated as a contributing factor to balance deficiencies. ${ }^{13,51,52}$ With weakness of intrinsic foot muscles there is a diminished ability to support the medial longitudinal arch. ${ }^{14}$ This leads to issues such as plantar fasciitis and medial tibial stress syndrome (shin splints), Achilles tendinopathy, posterior tibialis tendinopathy, anterior and posterior tibialis overuse. ${ }^{13,39}$ This can also work up the kinetic chain. Excessive pronation leads to internal rotation at the tibia which causes an increased valgus torque at the knee. ${ }^{4}$ Increased pronation causes the subtalar joint to evert which causes internal rotation of the tibia. This causes the femur to externally rotate which produces the valgus motion at the knee. ${ }^{53}$ These changes continue to move up the kinetic chain which is why the structure and function of the foot is critically important. ${ }^{54}$ Kelly et al. completed a study showing that 
activation of the quadratus plantae, flexor digitorum brevis, and abductor hallucis increases with an increase in postural demands. ${ }^{13}$

Tibialis posterior. The primary extrinsic muscle of the foot that is thought to support the medial longitudinal arch is the tibialis posterior (TP) ${ }^{6-10}$ Various studies have been done to learn the anatomy of the TP as well as what role it plays in supporting the foot during stance and gait. ${ }^{6,11,55}$ The tibialis posterior muscle has a vital role during gait as the primary dynamic stabilizer of the rear foot and the medial longitudinal arch. ${ }^{5,6,9,10,56}$ The muscle has primary roles of inversion at the subtalar joint and stabilizing the midfoot, it is also a foot adductor and plantarflexor. ${ }^{6,957,58}$ The TP has multiple insertions once it passes the medial malleolus and enters into the foot. The insertions are divided into two divisions referred to as superficial and deep. ${ }^{7}$ With the superficial component being divided into an anterior, medial and posterior aspect. ${ }^{9}$ The anterior aspect is the largest and attaches to the navicular tuberosity and the inferior surface of the medial cuneiform. ${ }^{7,34}$ The deep insertion has many insertions along the intermediate and lateral cuneiforms, the cuboid, and the $2^{\text {nd }}$ and $4^{\text {th }}$ metatarsal bones. ${ }^{7}$ The complex anatomy of the TP insertion sites serve to support the MLA. With dysfunction of the TP muscle stability of the foot is disrupted and can lead to a progressive FFD. The TP tendon along with the spring ligament is stretched in individuals with FFD, this results in a decrease in the height of the MLA. ${ }^{1}$ Studies have shown that the TP muscle is vital to keep the integrity and restore the MLA height during cyclic loading. ${ }^{8}$

Acquired pes planus is a popular chronic foot disorder noted by flattening of the medial longitudinal arch and decreased function of the posterior medial supporting tissues, mainly the tibialis posterior. ${ }^{5}$ The posterior tibialis is unable to lift the medial longitudinal arch and lock the mid tarsal joint. This causes an inability to stabilize the hind foot and increases risk of injury to 
other soft tissue structures. ${ }^{5}$ Studies have shown that dysfunction of the posterior tibialis during gait causes a posterior shift of the center of gravity and puts stress on the medial aspect of the foot. ${ }^{55}$ Imhauser et al. found that the function of the posterior tibialis during gait is to shift the center of pressure anteriorly and prevent the forces acting on the foot to shift medial, which causes a collapse of the medial longitudinal arch and eversion of the hind foot. ${ }^{55}$ Dysfunction of the posterior tibialis limited the ability of the muscle to perform both functions. It was found that the center of pressure would shift anteriorly, but the arch would still collapse. Studies found that individuals with posterior tibialis tendon dysfunction have an increased length of the posterior tibialis tendon. This showed that subjects with an increase in tendon length experience an increase in dysfunction of the tibialis posterior and the dynamic support of the medial longitudinal arch. ${ }^{59,60}$

Further research has been done to show that the tibialis posterior is essential to maintain medial longitudinal arch height during axial loading. ${ }^{8}$ Kamiya et al. study indicated that medial longitudinal arch height is not able to be maintained by passive support alone. It is necessary for intrinsic and extrinsic muscles to play a role in maintaining the stability and height of the medial longitudinal arch. Neville et al. ${ }^{57}$ found that individuals with posterior tibialis tendon dysfunction have an increased loading along the medial structures of the foot. The decrease in strength showed an increase in medial loading. These findings further support the idea that the tibialis posterior plays a vital role in supporting the medial longitudinal arch.

\section{Arch Deformities}

There are various deformities within the foot from the arches to the toes. The deformities in the arch can be localized to the medial longitudinal arch, specifically in reference to the height of the medial longitudinal arch and its effect on postural control in both static and dynamic 
function. The medial longitudinal arch has two extremes of structural position, high arch or pas cavus and low arch or pes planus. ${ }^{1}$

Individuals with pes cavus generally bear weight along the metatarsal heads and the lateral aspect of the foot or the $4^{\text {th }}$ and $5^{\text {th }}$ rays. With repeated pressure from weight bearing these lateral structures and the lateral aspect of the calcaneus develop bruising and calcifications due to adaptations under Wolff's law. With the high arches' inefficiency to distribute forces in weight bearing, one might develop a callus under the head of the $2^{\text {nd }}$ metatarsal. A high arched foot's inefficiency to distribute forces in weight bearing, one might develop a callus under the head of the 2 nd metatarsal. ${ }^{2}$ High arches produce a short plantar fascia which also can lead to a tight Achilles. With a tight plantar fascia the foot is unable to transfer forces and they move up the leg through the subtalar and talocrural joints. ${ }^{1}$ This leads to stress fractures in the lower extremity. ${ }^{1}$ Individuals with pes cavus will have an inverted subtalar joint. Tight inverter and plantar flexor muscles and weak everter muscles are often seen with pes cavus. ${ }^{1}$

Pes planus has been found to be the most common foot problem among adults in the United States. ${ }^{2}$ There is a direct correlation between activation of muscles of the foot and height of the medial longitudinal arch. The lower the medial longitudinal arch, the less activity of the muscles that support it. Lee et al. found that activation of the abductor hallucis was lower in subjects with pas planus compared to those with a neutral foot. ${ }^{47}$ With pes planus, the head of the talus sits more medial in the plantar direction relative to the navicular. This position of the talus stretches the calcanonavicular ligament and the tendon of the tibialis posterior. These two structures being put on a stretch compromises the height of the medial longitudinal arch. ${ }^{1}$ With pes planus not only is there a flattening of the medial longitudinal arch, but also rear foot valgus and abduction of the forefoot on the hind foot. ${ }^{34,45}$ The forefoot and hind foot structures untwist 
from each other causing an everted calcaneus and an abducted forefoot. This flattens the medial longitudinal arch and causes the "too many toes" phenomenon when inspecting an individual from posterior. ${ }^{1}$ When examining an individual's foot posture from behind the examiner should see the entire 5th digit and part of the $4^{\text {th }}$, if more toes are visible it is referred to as too many toes. Rigid flat foot is present when an individual has a flat foot both in sitting and in standing. Supple flat foot is when the individual has a visible medial longitudinal arch in sitting and when they stand up the arch flattens out. Individuals with a flat foot have shorter everter muscles, stretched inverter muscles and laxity of the medial ligaments of the foot. ${ }^{1}$

\section{Calcaneal Eversion in Relationship to Pronation}

Increased navicular drop independent or combined with increased calcaneal eversion have been thought to suggest a decrease in height of the medial longitudinal arch. ${ }^{61}$ Abnormal subtalar joint pronation may cause prolonged increased loads on other structures of the foot and may be evidenced by an increase in maximal calcaneal eversion. ${ }^{62-64}$ Arangio et al. found that as the calcaneus everts the borne load shifts from lateral to medial. Increasing the pressure put on the head of the first metatarsal and decreasing the load on the fifth. ${ }^{65}$ In weight bearing, forces are absorbed and transmitted through the subtalar joint. Specifically, the talus distributes forces to the navicular and calcaneus. ${ }^{1}$ In individuals with flat feet the talus plantar flexes and adducts causing a stretch on the spring ligament and putting pressure on the posterior tibialis tendon. ${ }^{66}$ All of this causes a decrease in the height of the medial longitudinal arch and forces the calcaneus also known as the hind foot to evert at the subtalar joint. ${ }^{1,67}$ When assessing abnormal pronation a measurement of calcaneal eversion greater than three degrees is thought to contribute to excessive pronation. ${ }^{68}$ Eversion at the subtalar joint is thought to decrease the rigidity of the foot during ambulation and is less capable to absorb ground forces. This causes an increase in 
activity of the intrinsic and extrinsic muscles of the foot to support the medial longitudinal arch which overtime produces fatigue of the muscles and various collateral injuries to the surrounding bony and soft tissue structures. ${ }^{1}$ Calcaneal eversion occurs at the subtalar joint. The subtalar joint is the articulation between the underside of the talus and the superior surface of the calcaneus. The articulation occurs at two separate facets, a posterior facet where the inferior concave surface of the talus rests on the superior convex surface of the calcaneus, and an anterior facet with a convex talar facet fitting into a concave calcaneal surface. ${ }^{63}$ These specific articulations allow for motions of the subtalar joint to occur in all three cardinal planes. These combined motions make up supination and pronation. Eversion, abduction and slight dorsiflexion of the foot are termed as pronation. Whereas inversion, adduction and plantarflexion of the foot is termed supination. ${ }^{63,69}$ These joint motions allow for efficient movement and transmission of forces. Subotnick quantified the total amount of normal subtalar joint motion that should occur is between twelve degrees of inversion and 6 degrees of eversion. The total 18 degrees of motion is normal for subtalar motion during movement. ${ }^{70}$

\section{Treatment of Pes Planus}

Intrinsic muscle strengthening/effects on navicular drop. Various studies have assessed the effect of intrinsic muscle strengthening on height of the medial longitudinal arch. ${ }^{14,15,39,47,54,71}$ Previous therapy protocols suggest the use of towel curling exercises can strengthen the muscles supporting the arch. However, the exercise targets extrinsic muscles, in this case the flexor digitorum longus. ${ }^{72}$ Short foot exercises have been found to activate intrinsic foot muscles and help form the medial longitudinal arch. ${ }^{73} \mathrm{Jung}$ et al. found that activity of the abductor hallucis was greater in that of subjects performing short foot exercise compared to those performing towel curl exercise. Furthermore, they found that muscle activity of the abductor 
hallucis increased in subjects performing short foot exercise in standing compared to those sitting. ${ }^{73}$ This supports Kelly's research that the recruitment of plantar intrinsic muscles increases as postural demand increases. ${ }^{13}$ Jung et al. used short foot exercise protocol while in standing to increase activation of the abductor hallucis longus. ${ }^{45}$ Goo et al. performed a similar study however they used toe spreading exercise while weight bearing compared to sitting. All studies found increased muscle activity when standing compared to sitting. ${ }^{74}$ Mulligan et al. used a progressive approach where subjects began the short foot exercise while sitting and as the study continued the subjects began to perform the exercise while standing. ${ }^{39}$

Sulowska et al. studied the effect of plantar short foot exercises on foot posture in long distance runners. They found that performing exercises to work the intrinsic muscles including Vele's forward lean and reverse tandem gait helped to modify foot posture and reduce pronation. ${ }^{54}$ The subjects in the study who performed the above exercises did not experience a significant change in calcaneal eversion compared to the group that performed the short foot exercise. When strengthening intrinsic foot muscles there needs to be a broad approach to the muscles being activated. Not all exercises activate the same muscles and produce the same changes in foot posture. It has been suggested that to get the most out of short foot exercises the subject should perform the exercises in subtalar neutral. McKeon et al. describes teaching subjects how to find subtalar neutral on their own before starting their protocol. ${ }^{3}$ Performing the exercises in subtalar neutral can help increase foot posture changes as muscle activation can increase with proper foot posture during exercise. ${ }^{75}$ Proper foot posture can improve single leg dynamic balance as shown in a study by moon et al. ${ }^{76}$ 
Tibialis posterior strengthening. Other researchers focus on the extrinsic foot muscles, or the prime movers such as the posterior tibialis muscle. The plethora of attachment sites the posterior tibialis has lends to its ability to support the medial longitudinal arch when standing and walking. ${ }^{11}$ The most common method that has been used to record posterior tibialis muscle activity is an EMG. ${ }^{9}$ Its ability to show muscle activation during movement makes it an invaluable tool. However there are relatively few studies that use intramuscular electrodes to record posterior tibialis activity. It is difficult to isolate the tibialis posterior with plantar flexion as the bulk of the plantarflexion is performed by the gastrocnemius and soleus complex. Therefore, studies have used multiple exercises as intervention for strengthening of the posterior tibialis. ${ }^{77}$ Kulig et al. ${ }^{58}$ used magnetic resonance imaging (MRI) to detect signal strength of the posterior tibialis during various movements. He used closed chain resisted foot adduction, unilateral heel raise, and open chain resisted supination (foot supination). ${ }^{58}$ Results showed signal increase with all three exercises with closed chain resisted foot adduction eliciting the greatest response from the MRI. With the unilateral heel raise the peroneus longus, soleus, and gastrocnemius were more active than the posterior tibialis. ${ }^{58}$ Still it is useful to perform this exercise as the posterior tibialis muscle is active during the movement. Bek et al. used what they described as posterior tibialis strengthening in conjunction with unilateral and bilateral heel raise in rehabilitation for individuals with posterior tibialis tendinopathy. ${ }^{5}$ Their study finds that both home based and supervised rehabilitation of the posterior tibialis improves muscle strength of the posterior tibialis. Kamiya et al. used cadaver legs to study the effect of posterior tibialis on the medial longitudinal arch during axial loading. Applying tension to the posterior tibialis in the experimental group and no tension in the control group showed that the active posterior tibialis tendon reduced the drop in the height of the navicular as cyclic loading increased. ${ }^{8}$ 


\section{Classification and Measurement}

In 1998 Menz conducted a review of the many various methods of clinically measuring foot pronation. ${ }^{20}$ Looking at arch height, footprint indices, valgus index, navicular drift and navicular drop he concluded that measurement of navicular drop and drift may prove to be the most applicable method for measuring foot pronation. However, at the time there was not much research conducted on use of the arch height index.

There are a few variables to consider when measuring and classifying foot posture. Height, weight, foot size, age and gender are common demographic variables of subjects in research. The pediatric foot is still developing and maturing compared to that of an adult foot. The majority of children are born flat footed and the arch develops over the first 5 to 10 years of a child's life. ${ }^{78}$ The prevalence of pes planus at age 3 was estimated to be $44 \%-68 \%$ and decreases to $21 \%-24 \%$ by age $6 .{ }^{79-82}$ Drefus et al. conducted a reliability study of the arch height index to measure foot posture in children. ${ }^{78}$ Finding that arch height index has good inter and intra-rater reliability when measuring pediatric patients. When looking at the effect of weight on changes in arch height Song et al. ${ }^{83}$ studied the effect of weight loss on foot structure and function in obese adults. Their study yielded results showing that weight loss decreases the magnitude of plantar pressure on the lateral arch of the foot. There were no significant differences between groups in regards to foot structure and arch height index. Indicating that weight does not have an effect on medial longitudinal arch height. Another study conducted by Nilsson et al. ${ }^{84}$ used body mass index (BMI) to classify weight and study the effect on maximum navicular height and navicular drop. There was no correlation in both of their measures supporting the thought that weight has a negligible effect on height of the medial longitudinal arch. Zifchock et al. ${ }^{85}$ conducted a study on the factors effecting lateral dominance, arch height, 
and arch stiffness. Among them they looked at effects of gender on the medial longitudinal arch. Their study found no significant differences between genders in arch height index. However, women do tend to have less stiffness of the medial longitudinal arch suggesting women may be at greater risk for soft tissue injuries compared to males. ${ }^{1}$

Arch indices. There are various ways to clinically assess foot posture disorders with static stance being the simple and minimally evasive way to measure, that produces a high level of consistency and validity. ${ }^{86}$ The method of measuring the height of the medial longitudinal arch has evolved over time with many variations used to perform similar measurements. Cowan et al. were one of the first to establish what is termed the bony arch index (BAI) which uses the ratio of total foot length to navicular height. ${ }^{87}$ Foot length is measured from the heel to the metatarsal phalangeal joint and navicular height is measured from the ground to the most prominent aspect of the navicular tubercle while the subject is fully bearing weight on the foot being measured. ${ }^{87}$ Although Cowan performed his measurements in full body weight other researchers have taken measurements in $50 \%$ or $90 \%$ body weight. Researchers have also defined foot length differently by specifying the use of the most posterior aspect of the heel to the first metatarsal phalangeal joint. ${ }^{86}$ The variations of these classifications and measurements make it difficult to establish validity and normative values using the bony arch index. The bony arch index has evolved in to the arch height index that various researchers have used to measure height of the medial longitudinal arch and classify foot posture. Originally calculated using the ratio of dorsum foot height at $50 \%$ of truncated foot length (measured from the most posterior aspect of the calcaneus to the first metatarsal phalangeal joint) this measurement made it possible to have normative values for the arch height index ${ }^{88}$ Another use for foot length is measuring from the most posterior aspect of the calcaneus to the tip of the longest toe. ${ }^{17,88,89}$ Reliability 
studies comparing both measurements for foot length have produced high to very high intra and inter-rater reliability. ${ }^{88,89}$ Williams and McClay ${ }^{88}$ found the most reliable valid measurements in their study were the dorsum height divided by truncated foot length rather than using the length of the entire foot.

Reliability studies for the arch height index have been performed by various researchers and their ICC numbers suggest very high reliability for both intra and inter rater. ${ }^{21,88}$ Richards et al ${ }^{89}$ produced high intra and inter rater reliability using the arch height index measurement system device. They established repeatable results and high reliability of the measurement device to validate the use of this versus the calipers originally used to measure arch height index. Williams and $\mathrm{McClay}^{88}$ originally used hand held digital calipers to measure arch height index. Their study produced high to very high intra and inter-rater reliability testing in both $10 \%$ and $90 \%$ weight bearing. The researchers chose $10 \%$ weight bearing because they saw the entire plantar surface of the foot in contact with the floor but the foot is still in a controlled weighted position. Comparing caliper measurements to measurements using the arch height index measurement system there is no discernable differences in reliability and validity of these measurements. Suggesting that using hand held devices if more expensive measures are not feasible will still produce valid results.

Navicular drop test. The navicular drop test is a popular method to assess medial longitudinal arch height. ${ }^{19}$ One of the first researches to assess changes in medial longitudinal arch height using navicular drop, Brody ${ }^{90}$ used the test to assess foot mobility and excessive pronation in runners. He conducted the measurement with the patient standing on a firm surface and their foot in subtalar neutral. The most prominent aspect of the navicular tuberosity is palpated and the height is marked on an index card. The patient is instructed to relax and the 
mark of the navicular height is made again. The difference was the relaxed position taken from the neutral position with a difference of $10 \mathrm{~mm}$ or less being classified as normal, with greater than $15 \mathrm{~mm}$ classified as abnormal. ${ }^{19,90}$ Brody did not provide any normative data in his study to justify the ranges he provided for normal and abnormal measurements. There were also no data to show reliability of measurements to support his use of the navicular drop test.

Studies have been done to classify the reliability of navicular drop and establish cut-off values in an effort to produce valid ranges to categorize various foot postures. ${ }^{84,91-93}$ Nilsson's study found that a normal navicular drop was 0.6 to $1.8 \mathrm{~cm}(6-18 \mathrm{~mm})$ which is in the range of Brody's original study listed above. Their study also found ranges of arch height on a scale ranging from severely low arch $(<2.7 \mathrm{~cm})$ to severely high $\operatorname{arch}(>6.4 \mathrm{~cm})$ and range of motion values classifying them from very flexible $(>2.3 \mathrm{~mm})$ of navicular drop) to very rigid $(<0.0 \mathrm{~mm}$ of navicular drop). Nilsson's range for a low arch was $1.8 \mathrm{~mm}$ to $2.3 \mathrm{~mm}$ of navicular drop from a subtalar neutral position measurement to a relaxed stance ${ }^{84}$ Many other researchers used a measurement of greater than $10 \mathrm{~mm}$ of navicular drop to classify a low arched, excessively pronated foot. ${ }^{16,76,94,95}$

Reliability studies have been done to look into intra tester and inter tester reliability levels of the navicular drop to establish the validity of the navicular drop test. ${ }^{91,93,96}$ Sell et al. established high intra tester ICC of 0.95 resting and 0.92 in subtalar neutral closed chain position as well as good results 0.83 when taking the difference between the two measurements. Sell et al. used experience testers in their study just as Ator et al. ${ }^{97}$ did previously. Vinicombe et al. ${ }^{91}$ found good intra tester reliability using the navicular drop test however they had a high level of variability in their study. Using inexperienced testers Picciano et al. ${ }^{96}$ produced poor $(0.61)$ to moderate (0.79) intra tester reliability when measuring navicular drop. Looking at these studies 
one can believe that measurement of navicular drop done by one experienced clinician can produce good to high reliability and minimal variability among measurements.

Plantar pressures. So far subjective measures of the medial longitudinal arch have been discussed; however a different way of measuring the medial longitudinal arch and weight bearing patterns in the foot is through radiographic imaging and plantar pressures. The repeatable and simplistic nature of footprint analysis is a popular method to measure the medial longitudinal arch. ${ }^{98}$ Different methods have been proposed for objective measurement of the medial longitudinal arch which can be categorized as either direct or indirect. Direct methods of measuring the medial longitudinal arch such as anthropometric measurements and radiographic imaging ${ }^{99,100}$ compared to indirect footprint and photo analyses ${ }^{101,102}$. These methods have recently been thought to have the flaw of being static measurements rather than dynamic imaging done through plantar pressures. Chu et al. ${ }^{103}$ were able to use static pressure measurements and correlate footprints with various arch heights of individuals from low to high. Indicating that pressure matching can give a classification of arch height.

Pedobarography is a newer method that allows clinicians to measure plantar pressures during dynamic loading of the foot and lower extremity. ${ }^{104}$ Yalcin et al. ${ }^{105}$ conducted a study comparing static and dynamic measurements using radiographic imaging against plantar pressure mapping. They found that both static and dynamic methods can be used to successfully measure the medial longitudinal arch. These findings disproved their own hypothesis that the measurements should be significantly different.

Validity of the TekScan MatScan was established by an independent study which showed the device to be highly accurate when compared to other commonly used plantar pressure measurement systems. ${ }^{106}$ Reliability of these measurements has been studied by Zammit et al. ${ }^{107}$ 
which conducted a study of the TekScan MatScan system measuring plantar forces and pressures during barefoot level walking. The authors found that the TekScan MatScan showed moderate to good reliability in measuring maximum force, peak pressure, and average pressure during dynamic walking tests. These results put the TekScan MatScan on the same level of reliability as other available plantar pressure measurement systems and suggest it can be used in research with valid results.

When taking into account the sum of previous information it is known that both intrinsic muscles and extrinsic muscles support the medial longitudinal arch. Researchers are able to use plantar pressures to both statically and dynamically measure the medial longitudinal arch. To the knowledge of this research team no studies have been conducted comparing extrinsic muscles to intrinsic muscles and how they support the medial longitudinal arch. Using both static and dynamic plantar pressure measurements as well as navicular drop measurement the goal is to better understand what muscles support the arch and when those said muscles are most active whether it be standing or moving. 


\section{CHAPTER III: METHODOLOGY}

\section{Participants}

Thirty-one recreationally physically active individuals between the ages of 18 and 25 were recruited for this study (Table 1). Participants included in the study were healthy, asymptomatic adults with clinically classified pes planus (navicular drop measurement of $11 \mathrm{~mm}$ or higher). ${ }^{88}$ Average navicular drop for each group can be referenced in table 1 . Three subjects were excluded citing previous history of lower extremity surgery to the leg being studied. Four subjects were excluded who did not meet the required criteria of $11 \mathrm{~mm}$ of navicular drop when measured at baseline. The remaining 24 subjects ( 14 females, 10 males; age $=21.04 \pm 1.68$ years, height $=172.2 \pm 9.74 \mathrm{~cm}$, mass $=74.67 \pm 18.56 \mathrm{~kg}$ ) were randomly placed into three groups divided among Intrinsic Strengthening (IS), Extrinsic Strengthening (ES), and a control group (CG). The control group received no intervention whereas the other two groups received specific exercise protocols in accordance with the muscle group being strengthened. Participants' self-reported their age (years), gender, height (m), mass (kg) at baseline. The clinician performing measurements was blinded to group allocation. Prior to beginning data collection, the Institutional Review Board approved the study. All participants provided written consent before they were allowed to participate in the study.

\section{Instrumentation}

Arch height index was measured in bilateral stance with the dominant foot in subtalar neutral and 50\% weight bearing in relaxed position using a digital caliper (Cen-tech Part No. 47257, Model 93293). Plantar pressures were recorded during static stance and level barefoot walking using the TekScan MobileMat ${ }^{\mathrm{TM}}$ system with FootMat Research Software version 7.0 (Tekscan, Inc., Boston, MA). The apparatus is comprised of a $0.76 \mathrm{~cm}$ thick floor mat with a 
48.7 x $44.7 \mathrm{~cm}$ sensing area). The MobileMat ${ }^{\mathrm{TM}}$ samples data at a frequency of $40 \mathrm{Hertz}(\mathrm{Hz})$. Navicular drop was measured marking on an index card and measuring in millimeters with a ruler.

\section{Procedures}

All measurements were taken by one clinician, a certified athletic trainer with seven years of clinical experience. Measurements of navicular drop, AHI, and plantar pressures were taken at baseline and following a four week intervention. The dominant foot of each participant was measured in two standing positions. The dominant leg was defined as the stance leg when kicking a ball. For navicular drop, participants were seated with heels flat on the floor legs shoulder width apart and hips and knees at $90^{\circ}$ of flexion. The clinician placed the ankle of the foot being measured in subtalar neutral. The most prominent aspect of the navicular tubercle was palpated and marked with a fine tip marker. That height was marked on an index card. The participant was then told to stand up and relax their feet. The most prominent aspect of the navicular tubercle was again palpated and marked with a fine tip marker. That height was marked on the same index card and the difference was measured in millimeters with a ruler. This measurement was recorded as the participant's navicular drop.

For the AHI measurement, participants were asked to stand with heels against the wall. In bilateral stance, the subject was asked to attempt to bear equal weight through both feet. ${ }^{86}$ The dominant foot was placed into subtalar neutral which was defined as equal palpation of the medial and lateral aspects of the talar head. ${ }^{108}$ The examining clinician located the medial aspect of the first metatarsal phalangeal joint (MTP) and marked it with a fine tip marker at the approximate center of the joint. The clinician measured from the end of the wall to the end of the great toe. This was the full foot measurement. The clinician then took the measurement from the 
posterior aspect of the heel (wall) to the approximate center of the first MTP. This provided the measure of truncated foot length. The second measurement was taken using a digital caliper from the top of the dorsum of the foot at $50 \%$ of the whole foot length. The AHI was then calculated using the dorsum height and the truncated foot length. ${ }^{21}$ Truncated foot length has very high intra and inter-rater reliability as a measurement and can be used to avoid accounting for toe deformities. ${ }^{86}$ This measure was performed three times and average of the three measurements was recorded for each participant.

For plantar pressure measurements, a baseline calibration measurement was taken while the participant was in a single-leg static stance on the MobileMat ${ }^{\mathrm{TM}}$. The participant was instructed to stand on the TekScan MobileMat ${ }^{\mathrm{TM}}$ with full weight maintained through the dominant foot. The participant was measured and allowed to stand an arm length away from a wall and use their finger-tips against the wall for balance while attempting to continue full weight bearing through their dominant foot. The participant stood for 5 seconds while the calibration measurement was recorded and the calibration procedures followed the manufacturer recommendations for walking trials on the mat. Calibration measurements were saved for each participant and used to standardize the data for each individual. A second calibration measurement was taken to mark the first MTP joint on the dominant foot. The participant started the two step walking protocol making sure the heel of their dominant foot struck the pressure mat. The participant was told to pause and balance on their dominant foot while the clinician placed pressure on the mat at the level of the previously marked first MTP. This calibration measurement was saved for each participant to standardize the midfoot area of each participant. Midfoot was operationally defined as the area from the posterior aspect of the heel to the level of the first MTP joint. This contact area was measured in square centimeters by drawing a box 
surrounding the midfoot. Each participant had a box drawn from the calibration and placed over the subsequent walking trials for the remainder of the study. The two step walking protocol was used to capture dynamic plantar pressures as it portrays similar retest reliability to the often used mid-gait protocol. ${ }^{109-111}$ The two step method requires striking the platform on the second step and is suggested to reproduce plantar force and pressure information that is meant to be a reproduction of foot function during gait. Trials were omitted and repeated if the plantar pressure recording was not properly positioned when striking the mat. Proper positioning was defined as striking the approximate center of the mat with each trial. Other trials were omitted if the subject paused on the mat during testing, or the participant did not continue to walk past the mat for more than two steps. Five trials for the dominant foot were recorded for each participant. ${ }^{107}$ These methods have previously been found to be reliable for assessing force and pressure data with the MobileMat ${ }^{\mathrm{TM}} .{ }^{112,113}$ Measures were completed pre- and post-intervention for all three groups. For each trial, the peak average pressure for the entire measurement was taken and used as the average contact area for gait cycle during that specific foot strike. Peak average pressure is a pre-set calculation that the foot mat software calculates for the user. The box from the dynamic calibration was then placed over each trial and the midfoot contact area was measured.

\section{Intervention}

For both groups receiving an intervention, an instructional handout specific to their intervention was given to the participant to take home. A research assistant explained and taught the exercises and was available to answer any questions in person and demonstrate exercises as well as make sure participants were performing the exercises properly throughout the study. Each participant was also given a log to track how often they performed their exercises. 
Intrinsic muscle strengthening. Participants in the IS group received a protocol focused on strengthening intrinsic muscles of the foot only. No other interventions were provided in conjunction with the strengthening program. The strengthening protocol consisted of three exercises performed once daily five days a week for four weeks. Participants were given the short foot exercise (SFE), toe yoga (TY) and Vele's forward lean (VFL). ${ }^{39,54}$ These three exercises are shown to increase muscle activity of the plantar intrinsic muscles, specifically the abductor hallucis. ${ }^{54,74}$ All exercises were performed in a single leg stance position with the exception of Vele's forward lean performed in bilateral stance. SFE and TSE were performed in single leg standing position for the purpose of increasing muscle activity due to increased postural demands. ${ }^{13}$ For standing exercises participants were given instructions to stand next to a wall or a table top for balance. For the SFE participants were instructed to elevate their MLA, shorten their feet in an anterior-posterior direction by trying to bring the head of their first metatarsal toward their heel without flexing the toes. The toes and heel need to remain on the ground during the exercise. ${ }^{45}$ Participants performed three sets of five repetitions holding each repetition for 5 seconds. There was a prescribed two minute rest in between each set. ${ }^{45}$ For the TSE participants were instructed to spread their toes outward and raise their heel off the ground while maintaining spread out toes. The subject then was told to lower their heel maintaining spread out toes and increasing the MLA. The participant held this position for 5 seconds and then relaxed. ${ }^{74}$ Participants performed three sets of five repetitions with a two minute rest in between sets. Participants who performed the VFL were instructed to stand with arms alongside the body with feet shoulder-width apart. Without lifting their heels from the floor they were told to lean as far forward as comfortably possible. ${ }^{54}$ Participants performed three sets of five repetitions 
holding each repetition for five seconds with a two minute rest in between each set. All exercises were instructed by the research assistant and proper performance was visually verified before the subject left the testing lab.

Extrinsic muscle strengthening. Participants in the ES group received a protocol focusing on strengthening of the tibialis posterior muscle. The strengthening protocol consisted of three exercises performed once daily five days a week for four weeks. The participants were instructed to perform closed chain resisted foot adduction, unilateral heel raise (heel raise), and open chain resisted foot supination. ${ }^{77}$ These exercises have been shown to have high to moderate activation of the tibialis posterior muscle. ${ }^{58}$ The foot adduction and foot supination exercises were performed using resistance provided by elastic bands (Green Theraband, heavy resistance, Thera-Band, Hygenic Corporation, Akron, $\mathrm{OH})$. Bands were provided at a length of three feet to each participant during pre-intervention measurements and exercise education. Each exercise was performed for three sets of 10 repetitions. For the foot adduction exercise, participants were seated with their knees maintained at a forearm's width length apart and knees flexed to $90^{\circ}$ with feet flat on the ground. The elastic band was tied in a loop and placed around the distal aspect of the foot around the metatarsal heads providing resistance in a lateral direction. The participant was advised to place the loop of the band around a table leg to provide resistance if they did not have somebody present to provide resistance for them. From an abducted position, the participant slid their foot into adduction and then returned to the starting position. The foot remains flat on the floor for the entire exercise. For foot supination, participants were seated with knee extended with the elastic band placed around the distal aspect of their foot around the metatarsal heads. The participant pulled the opposite end of the band toward the shoulder on the same side of the foot being exercised. The participant was instructed to plantar flex their foot and 
invert against the resistance and then returned to the starting position. The participant was instructed to complete the full range of motion. Participants who performed the heel raise were told to stand next to a wall or a table top to provide balance by touching with their fingertips without providing support. The participant was instructed to raise up on their toes (contralateral limb not touching the ground, the stance leg, or another object) and return their heel to the ground. The knee remains in full extension throughout the exercise. ${ }^{58}$ 


\section{CHAPTER IV: RESULTS}

\section{Statistical Analysis}

To account for variance in initial measurements for both navicular drop and plantarpressure area among participants, change scores were calculated by subtracting the preintervention value from the post-intervention value. To compare the effects of the intervention, three one-way ANOVAs were used to compare change scores for the 3 intervention groups. The alpha level was set a priori at $\mathrm{p}<0.05$. Tukey HSD post-hoc testing was used to determine significant differences between groups. Effect sizes were calculated by using Cohen's d and categorized as trivial $(\leq 0.20)$, small $(0.21$ to 0.49$)$, moderate $(0.50$ to 0.79$)$, and large $(\geq 0.80) .{ }^{114}$

\section{Results}

Subjects self-reported $100 \%$ compliance with performing their prescribed exercises during their 4 week intervention period. Participants were instructed to perform exercises 5 days a week for four weeks. However, when examining subject reported exercise logs there were slight deviations from this recommendation. Descriptive statistics for navicular drop and plantarpressure area are listed in Table 2. A significant difference between groups was found for the change in navicular drop, $\mathrm{F}(2,21)=9.22, \mathrm{p}=0.001$, but not for the change in plantar pressure area, $\mathrm{F}(2,21)=1.05, \mathrm{p}=0.37$. No significant difference was found between groups for arch height index, $\mathrm{F}(2,21)=1.54, \mathrm{p}=.238$. A one-way ANOVA showed a significant difference within the extrinsic group pre to post intervention ( $\mathrm{p}=0.001$, effect size $=1.17,95 \% \mathrm{CI}=0.11$ to 2.23 ). Table 3. Post hoc comparisons revealed a significant difference in navicular drop between the extrinsic and control group $(\mathrm{p}=0.001$, effect size $=2.15,95 \% \mathrm{CI}=0.92$ to 3.38$)$ and the extrinsic and intrinsic group ( $\mathrm{p}=0.03$, effect size $=1.31,95 \% \mathrm{CI}=0.23$ to 2.39 ), but no difference between the control and intrinsic group $(\mathrm{p}=0.31)$. Table 4. 
Table 1.

Descriptive Statistics for Demographic Data by Group

\begin{tabular}{cccccc}
\hline Group & Males & Females & Age (years) & Height $(\mathrm{cm})$ & Mass(kg) \\
\hline Extrinsic & 4 & 4 & $21.88 \pm 1.96$ & $170.4 \pm 5.70$ & $74.9 \pm 20.27$ \\
\hline Control & 3 & 5 & $20.88 \pm 1.64$ & $176.1 \pm 12.93$ & $80.16 \pm 19.61$ \\
\hline Intrinsic & 3 & 5 & $20.38 \pm 1.19$ & $170.2 \pm 9.31$ & $68.95 \pm 16.24$ \\
\hline Total & 10 & 14 & $21.04 \pm 1.68$ & $172.2 \pm 9.74$ & $74.67 \pm 18.56$ \\
\hline
\end{tabular}

Table 2.

Descriptive Statistics for Navicular Drop, Arch Height Index and Plantar-Pressure

\begin{tabular}{cllll}
\hline & & Extrinsic & Control & Intrinsic \\
\hline \multirow{2}{*}{$\begin{array}{c}\text { Navicular Drop } \\
(\mathrm{mm})\end{array}$} & Pre & $14.88 \pm 4.36$ & $14.38 \pm 4.78$ & $13.13 \pm 1.55$ \\
\cline { 2 - 5 } & Post & $9.50 \pm 4.84$ & $13.25 \pm 4.92$ & $10.50 \pm 2.93$ \\
\cline { 2 - 5 } & Change & $5.38 \pm 2.13^{*}$ & $1.13 \pm 1.81$ & $2.63 \pm 2.07$ \\
\hline \multirow{3}{*}{ AHI } & Pre & $0.358 \pm 0.034$ & $0.349 \pm .024$ & $0.358 \pm 0.020$ \\
\cline { 2 - 5 } & Post & $0.359 \pm 0.033$ & $0.360 \pm 0.027$ & $0.358 \pm 0.022$ \\
\cline { 2 - 5 } & Change & $0.001 \pm 0.013$ & $0.011 \pm 0.015$ & $0.000 \pm 0.014$ \\
\hline \multirow{2}{*}{$\begin{array}{c}\text { Plantar Pressure } \\
\text { (square cm) }\end{array}$} & Pre & $74.81 \pm 14.65$ & $82.35 \pm 21.55$ & $75.80 \pm 22.65$ \\
\cline { 2 - 5 } & Post & $72.85 \pm 15.13$ & $80.85 \pm 24.55$ & $75.95 \pm 23.00$ \\
\hline
\end{tabular}

*Significant difference from control and intrinsic group

Table 3 .

Effect Size for Group Differences Pre to Post Intervention of Navicular Drop

\begin{tabular}{lcccc}
\hline Group & Effect Size & $\begin{array}{c}\text { Std. Error of } \\
\text { Estimate }\end{array}$ & \multicolumn{2}{c}{$\begin{array}{c}\text { 95\% Confidence Interval } \\
\text { Lower-Upper }\end{array}$} \\
\hline Extrinsic & 1.17 & 0.54 & 0.11 & 2.23 \\
\hline Control & 0.23 & 0.50 & -0.75 & 1.22 \\
\hline Intrinsic & 0.54 & 1.12 & 0.07 & 2.17 \\
\hline
\end{tabular}


Table 4.

Effect Size for Between Group Differences Post Intervention

\begin{tabular}{lccc}
\hline \multicolumn{1}{c}{$\begin{array}{c}\text { Group } \\
\text { comparison }\end{array}$} & Effect Size & \multicolumn{2}{c}{$\begin{array}{c}\text { 95\% Confidence Interval } \\
\text { Lower-Upper }\end{array}$} \\
\hline $\begin{array}{l}\text { Extrinsic v } \\
\text { Control }\end{array}$ & 2.15 & 0.92 & 3.38 \\
\hline $\begin{array}{l}\text { Extrinsic v } \\
\text { Intrinsic }\end{array}$ & 1.31 & 0.23 & 2.39 \\
\hline
\end{tabular}




\section{CHAPTER V: DISCUSSION AND CONCLUSION}

\section{Discussion}

The purpose of this study was to investigate and compare the effect of strengthening the intrinsic versus extrinsic muscles of the foot and how this would affect navicular drop and plantar pressure area. Specifically, this study attempted to examine how specific exercises targeted for either the intrinsic or extrinsic muscles affected the support of the MLA. No previous research has compared both static and dynamic changes of the medial longitudinal arch post strengthening intervention between intrinsic and extrinsic muscle groups. Several studies have looked at changes in navicular drop or arch height index, however, no study has examined plantar pressure changes, specifically, focusing on changes in contact area of the midfoot during gait. These measurements can potentially provide clinicians with a better understanding at how the foot dynamically changes with certain exercises. Our study revealed significant changes in navicular drop following the intervention in individuals who performed extrinsic muscle strengthening exercises when compared to the control and intrinsic groups. Furthermore, these results showed large effect sizes.

Several studies have researched the effect of muscle training and/or fatigue on changes in navicular drop. ${ }^{14,39,71}$ These studies have looked at navicular drop as a static measurement not focusing on dynamic changes within the foot post exercise. Kim et al. ${ }^{71}$ found that SFE produced a significant decrease in navicular drop when comparing their outcomes to the use of arch support insoles. Similarly Mulligan et al. ${ }^{39}$ also found a significant decrease in navicular drop after a four week intervention of SFE. However Kim's study produced a $3.7 \mathrm{~mm}$ reduction in navicular drop over five weeks compared to only 2.2 in Mulligan's study over an 8 week period. Headlee et al. ${ }^{14}$ reversed course in his study using great toe curl exercises to prove that fatiguing 
the intrinsic muscles of the foot would increase navicular drop. His study produced a statistically significant navicular drop of $1.8 \mathrm{~mm}$ after a bout of exercise. All of these studies show that the intrinsic muscles play a role in supporting the MLA. However, in this study it was found that the TP plays a more significant role in supporting the MLA. The main variables tested in this research study were navicular drop for static measurements. Dynamic foot postures changes, specifically contact area of the midfoot, were taken with pressure mapping. Navicular drop has been researched in the past as a measure of the morphology of medial longitudinal arch. ${ }^{16}$ Many researchers used a measurement of greater than $10 \mathrm{~mm}$ of navicular drop to classify a low arched, excessively pronated foot. ${ }^{16,76,94,95}$

Weak intrinsic muscles have been thought to provide limited support of the MLA which can cause an increased strain on the plantar aponeurosis. ${ }^{12}$ This theory was supported by Headlee et al. ${ }^{14}$ whose study demonstrated an increased navicular drop brought on by fatigue of the intrinsic muscles of the foot. We theorized that strengthening these muscles would improve static foot posture by decreasing intrinsic muscle fatigue. This theory was supported by Mulligan et al. ${ }^{39}$ which found that a strengthening protocol aimed at the intrinsic foot muscles produced a significant reduction in navicular drop. Our study produced no significant results with changes in navicular drop when compared to strengthening of extrinsic foot muscles or the control group. While previous research has shown that intrinsic muscles play a role in supporting the medial longitudinal arch, ${ }^{14,39,40}$ our data suggests they may not be as important to maintaining the integrity of the MLA.

Given that the prime movers of the foot and ankle have the ability to produce greater force compared to smaller intrinsic muscles, it is likely these extrinsic muscles can provide substantial support to the MLA. The TP has several insertions within the foot, with the largest 
being on the navicular tuberosity. The tibialis posterior's variety of bony attachments lend to its ability to play an important role in MLA morphology and proper foot function. ${ }^{6}$ Support for this theory is shown by Kamiya et al. ${ }^{8}$ who found that the tibialis posterior plays an essential role in maintaining the height of the MLA. Imhauser et al. ${ }^{55}$ also found that when unloading the tibialis posterior there is a medial shift in plantar pressures during the gait cycle. The current study supported these studies with decreases of navicular drop following an exercise intervention aimed at strengthening the TP. When looking at tendon and ligamentous changes in individuals with pes planus, there are two common tissues that are stretched. In an over pronated foot, the talus is displaced medially which stretches the spring ligament and can lengthen the tibialis posterior tendon. ${ }^{1}$ A lengthened posterior tibialis tendon has decreased capacity to support the MLA which contributes to sustained pes planus deformity. These tissues changes, accompanied by pes planus, support the theory of tibialis posterior integrity being important to the structure and posture of the foot.

Studies continue to look at static measurements of MLA, without taking into consideration dynamic foot posture changes and if static exercises really cause any change in MLA morphology during the gait cycle. Fernandez et al. ${ }^{115}$ studied plantar pressures and contact area of individuals with cavus foot and compared them to normal foot posture. Their results showed that the plantar pressures were significantly reduced in individuals with pes cavus and an increased pressure was placed on the forefoot. While we looked at plantar pressures of individuals with pes planus the antithesis can still be theoretically applied. Individuals with pes planus should have greater contact area, specifically in the medial aspect of the midfoot. Our study did not produce significant changes in the contact area of the midfoot following either of the two interventions. Small changes in plantar pressure contact area occurred in both the control 
group and the extrinsic group, however not in the intrinsic group. Regardless, these recorded changes were not statistically significant. Dynamic changes in the foot may not have occurred because all exercises performed were in static or open chained positions.

Similar to integrating strength training into functional movement patterns, perhaps combining commonly used foot muscle strengthening protocols into gait training can produce dynamic changes in foot morphology. It is important to strengthen muscle groups, but it is also just as important to make sure the joint or body part is moving in a functional manner. Proper muscle firing patterns, movement patterns and neuromuscular education are important for functional movements.

\section{Limitations}

This study used a 4 week intervention to investigate the effects of strengthening protocols on changes in height of the MLA. Six or eight week interventions may produce more significant results. The main outcome criteria was focusing on the height of the MLA, however force production or changes in strength were not investigated post intervention. There was no true measure to verify the exercises strengthened the muscles groups they were targeting.

\section{Conclusions and Future Research}

Our results suggest that posterior tibialis strengthening protocols have a greater effect on MLA support and in decreasing navicular drop during static measurement. While intrinsic muscles did show improvement in MLA height, our findings did not reveal statistically significant changes. Furthermore, our results failed to demonstrate any changes in dynamic plantar pressure following either an extrinsic or intrinsic muscle strengthening protocol. Future research needs to examine how to support the structural integrity of the MLA during the gait cycle. 


\section{REFERENCES}

1. Franco AH. Pes cavus and pes planus. Analyses and treatment. Phys Ther. 1987;67(5):688-694.

2. Gould N, Schneider W, Ashikaga T. Epidemiological survey of foot problems in the continental United States: 1978-1979. Foot Ankle. 1980;1(1):8-10.

3. McKeon PO, Hertel J, Bramble D, Davis I. The foot core system: a new paradigm for understanding intrinsic foot muscle function. Br J Sports Med. 2015;49(5):290.

4. Coplan JA. Rotational motion of the knee: a comparison of normal and pronating subjects. J Orthop Sports Phys Ther. 1989;10(9):366-369.

5. Bek N, Simşek IE, Erel S, Yakut Y, Uygur F. Home-based general versus center-based selective rehabilitation in patients with posterior tibial tendon dysfunction. Acta Orthop Traumatol Turc. 2012;46(4):286-292.

6. Kaye RA, Jahss MH. Tibialis posterior: a review of anatomy and biomechanics in relation to support of the medial longitudinal arch. Foot Ankle. 1991;11(4):244247.

7. MARTIN BF. OBSERVATIONS ON THE MUSCLES AND TENDONS OF THE MEDIAL ASPECT OF THE SOLE OF THE FOOT. J Anat. 1964;98:437-453.

8. Kamiya T, Uchiyama E, Watanabe K, Suzuki D, Fujimiya M, Yamashita T. Dynamic effect of the tibialis posterior muscle on the arch of the foot during cyclic axial loading. Clin Biomech (Bristol, Avon). 2012;27(9):962-966.

9. Semple R, Murley GS, Woodburn J, Turner DE. Tibialis posterior in health and disease: a review of structure and function with specific reference to electromyographic studies. J Foot Ankle Res. 2009;2:24. 
10. Thordarson DB, Schmotzer H, Chon J, Peters J. Dynamic support of the human longitudinal arch. A biomechanical evaluation. Clin Orthop Relat Res. 1995(316):165-172.

11. Bloome DM, Marymont JV, Varner KE. Variations on the insertion of the posterior tibialis tendon: a cadaveric study. Foot Ankle Int. 2003;24(10):780-783.

12. Allen RH, Gross MT. Toe flexors strength and passive extension range of motion of the first metatarsophalangeal joint in individuals with plantar fasciitis. J Orthop Sports Phys Ther. 2003;33(8):468-478.

13. Kelly LA, Kuitunen S, Racinais S, Cresswell AG. Recruitment of the plantar intrinsic foot muscles with increasing postural demand. Clin Biomech (Bristol, Avon). 2012;27(1):46-51 .

14. Headlee DL, Leonard JL, Hart JM, Ingersoll CD, Hertel J. Fatigue of the plantar intrinsic foot muscles increases navicular drop. J Electromyogr Kinesiol. 2008;18(3):420425.

15. Hashimoto T, Sakuraba K. Strength training for the intrinsic flexor muscles of the foot: effects on muscle strength, the foot arch, and dynamic parameters before and after the training. J Phys Ther Sci. 2014;26(3):373-376.

16. Mueller MJ, Host JV, Norton BJ. Navicular drop as a composite measure of excessive pronation. J Am Podiatr Med Assoc. 1993;83(4):198-202.

17. Weimar WH, Shroyer JF. Arch height index normative values of college-aged women using the arch height index measurement system. J Am Podiatr Med Assoc. 2013;103(3):213-217. 
18. Hillstrom HJ, Song J, Kraszewski AP, et al. Foot type biomechanics part 1: structure and function of the asymptomatic foot. Gait Posture. 2013;37(3):445-451.

19. McPoil TG, Cornwall MW, Medoff L, Vicenzino B, Forsberg K, Hilz D. Arch height change during sit-to-stand: an alternative for the navicular drop test. J Foot Ankle Res. 2008;1(1):3.

20. Menz HB. Alternative techniques for the clinical assessment of foot pronation. $J \mathrm{Am}$ Podiatr Med Assoc. 1998;88(3):119-129.

21. Butler RJ, Hillstrom H, Song J, Richards CJ, Davis IS. Arch height index measurement system: establishment of reliability and normative values. $J$ Am Podiatr Med Assoc. 2008;98(2):102-106.

22. Lynn SK, Padilla RA, Tsang KK. Differences in static- and dynamic-balance task performance after 4 weeks of intrinsic-foot-muscle training: the short-foot exercise versus the towel-curl exercise. J Sport Rehabil. 2012;21(4):327-333.

23. Jenkins DB. Hollinshead's Functional Anatomy of the Limbs and Back. Ninth ed2009.

24. Kido M, Ikoma K, Imai K, Tokunaga D, Inoue N, Kubo T. Load response of the medial longitudinal arch in patients with flatfoot deformity: in vivo 3D study. Clin Biomech (Bristol, Avon). 2013;28(5):568-573.

25. Kapandji A. The Physiology of the Joints: Lower Limb. Vol 2. Baltimore, MD: Williams and Wilkins; 1970.

26. Kessler R, Hertling D. Management of Common Musculoskeletal Disorders: Physical Therapy Principles and Methods. Philadelphia, PA: Harper \& Row, Publishers Inc; 1983. 
27. Tong JW, Kong PW. Association between foot type and lower extremity injuries: systematic literature review with meta-analysis. J Orthop Sports Phys Ther. 2013;43(10):700-714.

28. Dahle LK, Mueller MJ, Delitto A, Diamond JE. Visual assessment of foot type and relationship of foot type to lower extremity injury. J Orthop Sports Phys Ther. 1991;14(2):70-74.

29. Gabel CP, Melloh M, Burkett B, Michener LA. Lower limb functional index: development and clinimetric properties. Phys Ther. 2012;92(1):98-110.

30. Mei-Dan O, Kahn G, Zeev A, et al. The medial longitudinal arch as a possible risk factor for ankle sprains: a prospective study in 83 female infantry recruits. Foot Ankle Int. 2005;26(2):180-183.

31. McKENZIE J. The foot as a half-dome. Br Med J. 1955;1(4921):1068-1069.

32. Borton DC, Saxby TS. Tear of the plantar calcaneonavicular (spring) ligament causing flatfoot. A case report. J Bone Joint Surg Br. 1997;79(4):641-643.

33. Huang CK, Kitaoka HB, An KN, Chao EY. Biomechanical evaluation of longitudinal arch stability. Foot Ankle. 1993;14(6):353-357.

34. Pinney SJ, Lin SS. Current concept review: acquired adult flatfoot deformity. Foot Ankle Int. 2006;27(1):66-75.

35. Davis WH, Sobel M, DiCarlo EF, et al. Gross, histological, and microvascular anatomy and biomechanical testing of the spring ligament complex. Foot Ankle Int. 1996;17(2):95-102.

36. Daly PJ, Kitaoka HB, Chao EY. Plantar fasciotomy for intractable plantar fasciitis: clinical results and biomechanical evaluation. Foot Ankle. 1992;13(4):188-195. 
37. Deland JT, Arnoczky SP, Thompson FM. Adult acquired flatfoot deformity at the talonavicular joint: reconstruction of the spring ligament in an in vitro model. Foot Ankle. 1992;13(6):327-332.

38. HICKS JH. The mechanics of the foot. II. The plantar aponeurosis and the arch. $J$ Anat. 1954;88(1):25-30.

39. Mulligan EP, Cook PG. Effect of plantar intrinsic muscle training on medial longitudinal arch morphology and dynamic function. Man Ther. 2013;18(5):425-430.

40. Fiolkowski P, Brunt D, Bishop M, Woo R, Horodyski M. Intrinsic pedal musculature support of the medial longitudinal arch: an electromyography study. J Foot Ankle Surg. 2003;42(6):327-333.

41. Soysa A, Hiller C, Refshauge K, Burns J. Importance and challenges of measuring intrinsic foot muscle strength. $J$ Foot Ankle Res. 2012;5(1):29.

42. Alanen AM, Falck B, Kalimo H, Komu ME, Sonninen VH. Ultrasound, computed tomography and magnetic resonance imaging in myopathies: correlations with electromyography and histopathology. Acta Neurol Scand. 1994;89(5):336-346.

43. Kura H, Luo ZP, Kitaoka HB, An KN. Quantitative analysis of the intrinsic muscles of the foot. Anat Rec. 1997;249(1):143-151.

44. H. G. Gray's Anatomy. 37 ed. Linvingstone: Edinburgh; 1989.

45. Jung DY, Koh EK, Kwon OY. Effect of foot orthoses and short-foot exercise on the cross-sectional area of the abductor hallucis muscle in subjects with pes planus: a randomized controlled trial. J Back Musculoskelet Rehabil. 2011;24(4):225-231.

46. Wong YS. Influence of the abductor hallucis muscle on the medial arch of the foot: a kinematic and anatomical cadaver study. Foot Ankle Int. 2007;28(5):617-620. 
47. Lee JH, Cynn HS, Yoon TL, Choi SA, Kang TW. Differences in the angle of the medial longitudinal arch and muscle activity of the abductor hallucis and tibialis anterior during sitting short-foot exercises between subjects with pes planus and subjects with neutral foot. J Back Musculoskelet Rehabil. 2016.

48. Gray EG, Basmajian JV. Electromyography and cinematography of leg and foot ("normal" and flat) during walking. Anat Rec. 1968;161(1):1-15.

49. MANN R, INMAN VT. PHASIC ACTIVITY OF INTRINSIC MUSCLES OF THE FOOT. J Bone Joint Surg Am. 1964;46:469-481.

50. J B, C D. Muscles Alive: Their functions revealed by electromyography. Baltimore, MD: Williams and Wilkins; 1985.

51. Menz HB, Morris ME, Lord SR. Foot and ankle characteristics associated with impaired balance and functional ability in older people. J Gerontol A Biol Sci Med Sci. 2005;60(12):1546-1552.

52. Mickle KJ, Munro BJ, Lord SR, Menz HB, Steele JR. ISB Clinical Biomechanics Award 2009: toe weakness and deformity increase the risk of falls in older people. Clin Biomech (Bristol, Avon). 2009;24(10):787-791.

53. Subotnick SI. The foot and its relationship to gait: a series of articles and editorial comments. J Orthop Sports Phys Ther. 1980;2(2):48-54.

54. Sulowska I, Oleksy L, Mika A, Bylina D, Sołtan J. The Influence of Plantar Short Foot Muscle Exercises on Foot Posture and Fundamental Movement Patterns in LongDistance Runners, a Non-Randomized, Non-Blinded Clinical Trial. PLoS One. 2016;11(6):e0157917. 
55. Imhauser CW, Siegler S, Abidi NA, Frankel DZ. The effect of posterior tibialis tendon dysfunction on the plantar pressure characteristics and the kinematics of the arch and the hindfoot. Clin Biomech (Bristol, Avon). 2004;19(2):161-169.

56. BASMAJIAN JV, STECKO G. THE ROLE OF MUSCLES IN ARCH SUPPORT OF THE FOOT. J Bone Joint Surg Am. 1963;45:1184-1190.

57. Neville C, Flemister AS, Houck J. Total and distributed plantar loading in subjects with stage II tibialis posterior tendon dysfunction during terminal stance. Foot Ankle Int. 2013;34(1):131-139.

58. Kulig K, Burnfield JM, Requejo SM, Sperry M, Terk M. Selective activation of tibialis posterior: evaluation by magnetic resonance imaging. Med Sci Sports Exerc. 2004;36(5):862-867.

59. Neville C, Flemister A, Tome J, Houck J. Comparison of changes in posterior tibialis muscle length between subjects with posterior tibial tendon dysfunction and healthy controls during walking. J Orthop Sports Phys Ther. 2007;37(11):661669.

60. Johnson KA, Strom DE. Tibialis posterior tendon dysfunction. Clin Orthop Relat Res. 1989(239):196-206.

61. Brantingham JW, Adams KJ, Cooley JR, Globe D, Globe G. A single-blind pilot study to determine risk and association between navicular drop, calcaneal eversion, and low back pain. J Manipulative Physiol Ther. 2007;30(5):380-385.

62. Donatelli RA. Abnormal biomechanics of the foot and ankle. J Orthop Sports Phys Ther. 1987;9(1):11-16. 
63. Brown LP, Yavorsky P. Locomotor biomechanics and pathomechanics: a review. $J$ Orthop Sports Phys Ther. 1987;9(1):3-10.

64. Tiberio D. Pathomechanics of structural foot deformities. Phys Ther. 1988;68(12):18401849.

65. Arangio GA, Phillippy DC, Xiao D, Gu WK, Salathe EP. Subtalar pronation--relationship to the medial longitudinal arch loading in the normal foot. Foot Ankle Int. 2000;21(3):216-220.

66. Joseph J. A Textbook of Regional Anatomy. Baltimore, MD: University Park Press; 1982.

67. Hoppenfeld S. Physical Examination of the Spine and Extremities. New York, NY: Appleton-Century-Crofts; 1976.

68. Genova JM, Gross MT. Effect of foot orthotics on calcaneal eversion during standing and treadmill walking for subjects with abnormal pronation. J Orthop Sports Phys Ther. 2000;30(11):664-675.

69. JT M. Movements of the subtalar and transverse tarsal joints. The Anatomical record. 1941;80(4):397.

70. Subotnick S. Biomechanics of the Subtalar and Midtarsal Joints. Journal of the American Podiatry Association. 1975;65(8):9.

71. Kim EK, Kim JS. The effects of short foot exercises and arch support insoles on improvement in the medial longitudinal arch and dynamic balance of flexible flatfoot patients. J Phys Ther Sci. 2016;28(11):3136-3139.

72. Saeki J, Tojima M, Torii S. Clarification of functional differences between the hallux and lesser toes during the single leg stance: immediate effects of conditioning 
contraction of the toe plantar flexion muscles. J Phys Ther Sci. 2015;27(9):27012704.

73. Jung DY, Kim MH, Koh EK, Kwon OY, Cynn HS, Lee WH. A comparison in the muscle activity of the abductor hallucis and the medial longitudinal arch angle during toe curl and short foot exercises. Phys Ther Sport. 2011;12(1):30-35.

74. Goo YM, Heo HJ, An DH. EMG Activity of the Abductor Hallucis Muscle during Foot Arch Exercises Using Different Weight Bearing Postures. J Phys Ther Sci. 2014;26(10):1635-1636.

75. McKeon PO, Fourchet F. Freeing the foot: integrating the foot core system into rehabilitation for lower extremity injuries. Clin Sports Med. 2015;34(2):347-361.

76. Moon DC, Kim K, Lee SK. Immediate Effect of Short-foot Exercise on Dynamic Balance of Subjects with Excessively Pronated Feet. J Phys Ther Sci. 2014;26(1):117-119.

77. Houck J, Neville C, Tome J, Flemister A. Randomized Controlled Trial Comparing Orthosis Augmented by Either Stretching or Stretching and Strengthening for Stage II Tibialis Posterior Tendon Dysfunction. Foot Ankle Int. 2015;36(9):10061016.

78. Drefus LC, Kedem P, Mangan SM, Scher DM, Hillstrom HJ. Reliability of the Arch Height Index as a Measure of Foot Structure in Children. Pediatr Phys Ther. 2017;29(1):83-88.

79. Lin CJ, Lai KA, Kuan TS, Chou YL. Correlating factors and clinical significance of flexible flatfoot in preschool children. J Pediatr Orthop. 2001;21(3):378-382. 
80. Pfeiffer M, Kotz R, Ledl T, Hauser G, Sluga M. Prevalence of flat foot in preschool-aged children. Pediatrics. 2006;118(2):634-639.

81. Chen KC, Yeh CJ, Tung LC, Yang JF, Yang SF, Wang CH. Relevant factors influencing flatfoot in preschool-aged children. Eur J Pediatr. 2011;170(7):931-936.

82. Chen KC, Tung LC, Yeh CJ, Yang JF, Kuo JF, Wang CH. Change in flatfoot of preschool-aged children: a 1-year follow-up study. Eur J Pediatr. 2013;172(2):255-260.

83. Song J, Kane R, Tango DN, et al. Effects of weight loss on foot structure and function in obese adults: a pilot randomized controlled trial. Gait Posture. 2015;41(1):86-92.

84. Nilsson MK, Friis R, Michaelsen MS, Jakobsen PA, Nielsen RO. Classification of the height and flexibility of the medial longitudinal arch of the foot. J Foot Ankle Res. $2012 ; 5: 3$

85. Zifchock RA, Davis I, Hillstrom H, Song J. The effect of gender, age, and lateral dominance on arch height and arch stiffness. Foot Ankle Int. 2006;27(5):367-372.

86. McPoil TG, Cornwall MW, Vicenzino B, et al. Effect of using truncated versus total foot length to calculate the arch height ratio. Foot (Edinb). 2008;18(4):220-227.

87. Cowan DN, Jones BH, Robinson JR. Foot morphologic characteristics and risk of exercise-related injury. Arch Fam Med. 1993;2(7):773-777.

88. Williams DS, McClay IS. Measurements used to characterize the foot and the medial longitudinal arch: reliability and validity. Phys Ther. 2000;80(9):864-871.

89. Richards C, Card K, Song J, Hillstrom H, Butler R, Davis I. A Novel Arch Height Index Measurment System (AHIMS): Intra- and Inter-Rater Reliability. In. Book of 
Abstracts. Toledo Ohio: The Annual Meeting of the American Society of Biomechanics; 2003.

90. Brody DM. Techniques in the evaluation and treatment of the injured runner. Orthop Clin North Am. 1982;13(3):541-558.

91. Vinicombe A, Raspovic A, Menz HB. Reliability of navicular displacement measurement as a clinical indicator of foot posture. J Am Podiatr Med Assoc. 2001;91(5):262268.

92. Williams DS, McClay IS, Hamill J. Arch structure and injury patterns in runners. Clin Biomech (Bristol, Avon). 2001;16(4):341-347.

93. Sell KE, Verity TM, Worrell TW, Pease BJ, Wigglesworth J. Two measurement techniques for assessing subtalar joint position: a reliability study. $J$ Orthop Sports Phys Ther. 1994;19(3):162-167.

94. Shrader JA, Popovich JM, Gracey GC, Danoff JV. Navicular drop measurement in people with rheumatoid arthritis: interrater and intrarater reliability. Phys Ther. 2005;85(7):656-664.

95. Brantingham JW, Lee Gilbert J, Shaik J, Globe G. Sagittal plane blockage of the foot, ankle and hallux and foot alignment-prevalence and association with low back pain. J Chiropr Med. 2006;5(4):123-127.

96. Picciano AM, Rowlands MS, Worrell T. Reliability of open and closed kinetic chain subtalar joint neutral positions and navicular drop test. J Orthop Sports Phys Ther. 1993;18(4):553-558. 
97. Ator R, Gunn K, McPoil TG, Knecht HG. The Effect of Adhesive Strapping on Medial Longitudinal Arch Support before and after Exercise. J Orthop Sports Phys Ther. 1991;14(1):18-23.

98. Cavanagh PR, Rodgers MM. The arch index: a useful measure from footprints. $J$ Biomech. 1987;20(5):547-551.

99. Staheli LT, Chew DE, Corbett M. The longitudinal arch. A survey of eight hundred and eighty-two feet in normal children and adults. J Bone Joint Surg Am. 1987;69(3):426-428.

100. Viladot A. Surgical treatment of the child's flatfoot. Clin Orthop Relat Res. 1992(283):34-38.

101. Volpon JB. Footprint analysis during the growth period. J Pediatr Orthop. 1994;14(1):83-85.

102. Saltzman CL, Nawoczenski DA, Talbot KD. Measurement of the medial longitudinal arch. Arch Phys Med Rehabil. 1995;76(1):45-49.

103. Chu WC, Lee SH, Chu W, Wang TJ, Lee MC. The use of arch index to characterize arch height: a digital image processing approach. IEEE Trans Biomed Eng. 1995;42(11):1088-1093.

104. Skopljak A, Muftic M, Sukalo A, Masic I, Zunic L. Pedobarography in diagnosis and clinical application. Acta Inform Med. 2014;22(6):374-378.

105. Yalcin N, Esen E, Kanatli U, Yetkin H. Evaluation of the medial longitudinal arch: a comparison between the dynamic plantar pressure measurement system and radiographic analysis. Acta Orthop Traumatol Turc. 2010;44(3):241-245. 
106. Giacomozzi C. Appropriateness of plantar pressure measurement devices: a comparative technical assessment. Gait Posture. 2010;32(1):141-144.

107. Zammit GV, Menz HB, Munteanu SE. Reliability of the TekScan MatScan(R) system for the measurement of plantar forces and pressures during barefoot level walking in healthy adults. $J$ Foot Ankle Res. 2010;3:11.

108. Nielsen RG, Rathleff MS, Simonsen OH, Langberg H. Determination of normal values for navicular drop during walking: a new model correcting for foot length and gender. J Foot Ankle Res. 2009;2:12.

109. Bryant A, Singer K, Tinley P. Comparison of the reliability of plantar pressure measurements using the two-step and midgait methods of data collection. Foot Ankle Int. 1999;20(10):646-650.

110. Oladeji O, Stackhouse C, Gracely E, Orlin M. Comparison of the two-step and midgait methods of plantar pressure measurement in children. J Am Podiatr Med Assoc. 2008;98(4):268-277.

111. Bus SA, de Lange A. A comparison of the 1-step, 2-step, and 3-step protocols for obtaining barefoot plantar pressure data in the diabetic neuropathic foot. Clin Biomech (Bristol, Avon). 2005;20(9):892-899.

112. Van der Leeden M, Dekker JH, Siemonsma PC, Lek-Westerhof SS, Steultjens MP. Reproducibility of plantar pressure measurements in patients with chronic arthritis: a comparison of one-step, two-step, and three-step protocols and an estimate of the number of measurements required. Foot Ankle Int. 2004;25(10):739-744. 
113. Hughes J, Pratt L, Linge K, Clark P, Klenerman L. Reliability of pressure measurements: the EM ED F system. Clin Biomech (Bristol, Avon). 1991;6(1):14-18.

114. J. C. Statistical Power Analysis for the Behavioral Sciences. 2nd ed. Hillsdale, NJ: Lawrence Earlbaum Associates; 1988.

115. Fernández-Seguín LM, Diaz Mancha JA, Sánchez Rodríguez R, Escamilla Martínez E, Gómez Martín B, Ramos Ortega J. Comparison of plantar pressures and contact area between normal and cavus foot. Gait Posture. 2014;39(2):789-792. 


\section{APPENDIX A: EXERCISE HANDOUT}

Below you will find instructions on how to perform the exercises corresponding to the group you have randomly been assigned to. If you are in group 1 you are to perform the intrinsic foot muscle protocol. If you are in group two you are to perform the extrinsic foot muscle protocol. *All exercises performed in a single leg standing position will be performed on your "dominant leg" this was established in your initial visit with the research investigator. Your "dominant leg" is the leg you stand on when kicking a ball.

\section{Group 1}

\section{Exercise 1: Short foot exercise}

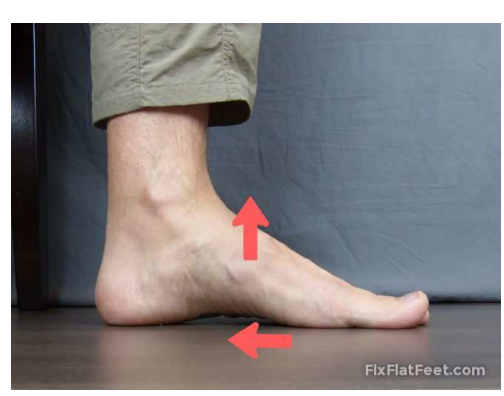

In a single leg standing position attempt to bring your big toe to your heel without curling your toes. You are literally attempting to shorten your foot to increase the height of the arch of your foot. You should feel the pressure of your big toe against the floor increase. Hold each attempt for 5 seconds and then relax. Perform 3 sets of 15 repetitions.

\section{Exercise 2: Forward Lean}

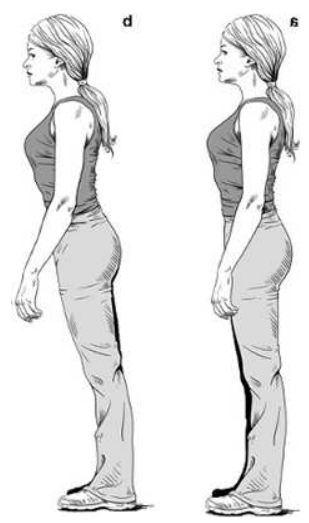

Begin by standing behind a chair with the back of the chair facing you. Stand with feet approximately shoulder width apart. Attempt to lean forward as far as you comfortably can while keeping your heels on the ground. You may use the chair to catch you if you lean to far forward, however try to not use the chair to balance yourself. Hold your lean for 5 seconds and then relax. Perform 3 sets of 15 repetitions.

\section{Exercise 3: Toe Yoga}

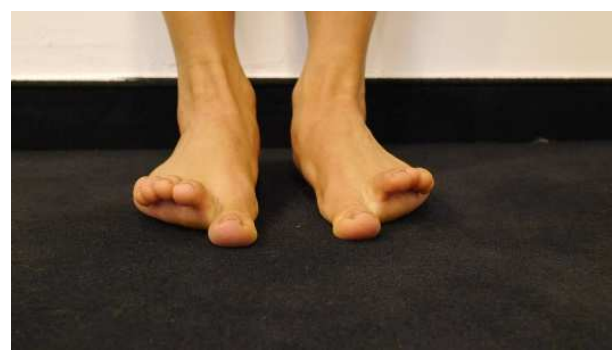

In a standing position with feet approximately shoulder width apart, attempt to lift your big toe while holding your other four toes on the floor. Next push your big toe into the floor and attempt to lift your other four toes off the floor simultaneously. This completes one repetition. Complete 3 sets of 15 repetitions. 
Below you will find instructions on how to perform the exercises corresponding to the group you have randomly been assigned to. If you are in group 1 you are to perform the intrinsic foot muscle protocol. If you are in group two you are to perform the extrinsic foot muscle protocol.

\section{Group 2}

Tie the green exercises band provided to you in a loop.

\section{Exercise 1: Foot Adduction}
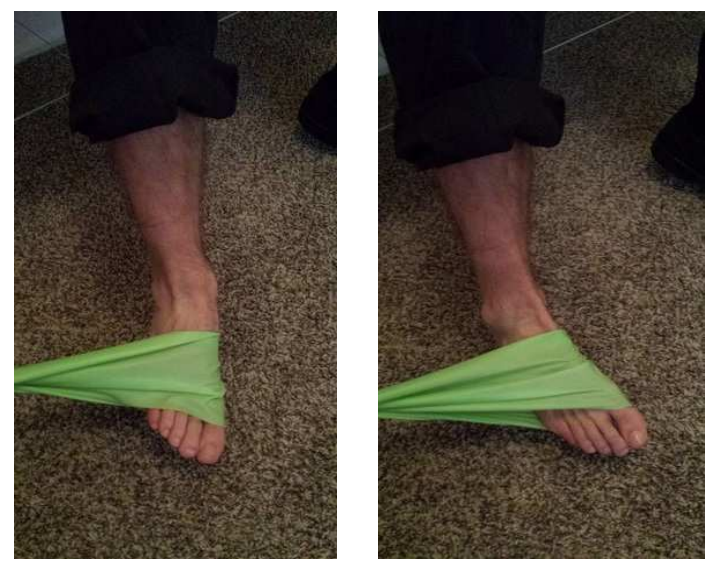

In a seated position with your feet flat on the floor and legs shoulder width apart. Place the band around the ball of your foot (just before your toes). Place the other end to a fixed point (example: around a table leg). Position yourself so the band is pulling your foot out (away from your body). Make sure the band is at full tension before you begin your exercise. While keeping your foot flat on the floor slide your foot so that you are moving your big toe towards the other stationary foot. Hold this for 5 seconds and then relax. Perform 3 sets of 15 repetitions.

\section{Exercise 2: Foot Inversion (supination)}

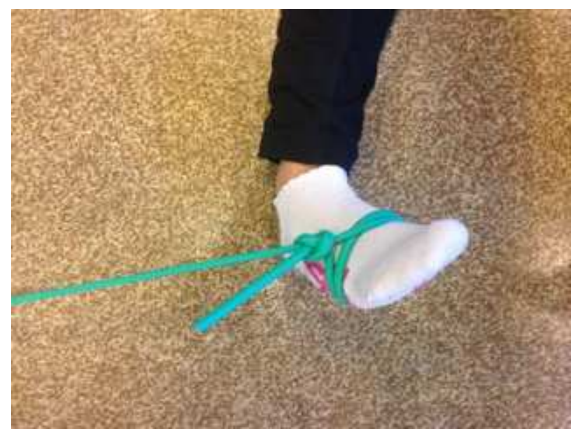

In a seated position place the foot not to be exercises flat on the floor. Place the foot to be exercised on a chair or stool of similar height. Keep your leg straight and loop the band around your foot just below your toes around the ball of your foot. Pull the band towards the same shoulder as the foot you are exercising. Push your foot down and inward, bringing your big toe towards the opposite leg and hold this for 5 seconds. Perform 3 sets of 15 repetitions. 


\section{Exercise 3: Single Leg Heel Raise}

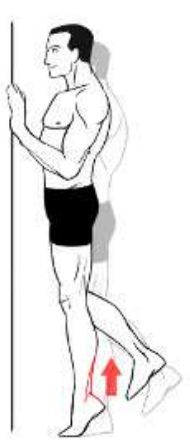

While standing transfer your weight fully to the foot being exercised with the opposite leg not touching the ground. Stand an arm length away from a wall so you may use your finger tips to balance. Raise your heel off the ground so you are on your toes. Slowly lower your heel back to the ground. Perform 3 sets of 15 repetitions. 